\title{
Distributed Model Predictive Control for the Atmospheric and Vacuum Distillation Towers in a Petroleum Refining Process
}

\author{
Shuzhan Zhang, Dongya Zhao * \\ College of Chemical Engineering, \\ China University of Petroleum (Hua Dong) \\ Shandong Qingdao, 266580, China \\ Email: SZ_8866@163.com,dyzhao@upc.edu.cn \\ *Corresponding Author
}

\author{
Sarah K. Spurgeon, Xinggang Yan \\ School of Engineering \& Digital Arts, \\ University of Kent \\ Canterbury, Kent CT2 7NT, United Kingdom \\ Email: S.K.Spurgeon@kent.ac.uk,X.Yan@kent.ac.uk
}

\begin{abstract}
This paper develops a distributed model predictive control strategy for the atmospheric and vacuum distillation tower, which constitutes a key process involved in refining petroleum. When considering an MPC implementation, it is known that computational complexity can be reduced if the system is first decomposed into multiple smaller dimensional subsystems. Optimally exploiting the modern computer networks available in industry, a distributed model predictive control implementation is developed for the atmospheric and vacuum tower system, which is assumed to be part of a wider petroleum refining process comprised of a number of sub-systems connected in series. For each subsystem, given the availability of mutual communication channels between subsystems and by using an iterative calculation approach, it will be seen that Nash optimality can be achieved. A low-cost solution that is readily implementable online is seen to achieve the control objective. The effectiveness of the approach presented in the paper is validated by the results of nonlinear simulation experiments.
\end{abstract}

Keywords-Distributed model predictive control; atmospheric and vacuum distillation tower; subsystem.

\section{INTRODUCTION}

Distillation is an important step in the oil refinery process within the petrochemical industry, and the atmospheric and vacuum tower is a typical subsystem involved in this process. The petrochemical industry is becoming increasingly largescale and the need for energy efficiency and increased productivity is becoming more prominent. This quest to improve economic benefits has resulted in increasing demands on the control performance of the tower. The atmospheric and vacuum tower system is, however, complex with many uncertain parameters. Changes in these parameters can cause system instability and there is thus a need to consider the development of robust control strategies whilst accommodating the need for economically viable solutions.

Within the control system, the control loops are closed using real-time communication networks and thus belong to a class of networked control systems (NCSs) [1]. Such NCSs are widely investigated within industry because of the advances in control design, flexibility and reduction in costs that they can underpin [2]. However, the resulting industrial systems become more and more complex for the purposes of system analysis and controller design [3]. It is not straightforward to control these systems with a single centralised MPC control structure because of the computational complexity and communication bandwidth limitations [4].

Several approaches have therefore been developed to deal with the problem of computational complexity within MPC. Shekhar and Maciejowski [5] introduced a new formulation of variable horizon MPC that utilised move locking for reducing computational complexity. Ling et al. [6] proposed a form of MPC in which the control variables were moved asynchronously. The computation time was reduced by solving the MPC problem for each subsystem sequentially. A parameterised field-programmable gate array implementation of a customised quadratic programming solver for optimal control of linear processes with constraints was presented in [7]. A new MPC formulation was introduced by taking advantage of novel computational opportunities. Rakovic et al. [8] developed a parameterised tube MPC synthesis method, which was computationally efficient while it possessed rather strong system theoretical properties.

The advantages of MPC have begun to play an important role in the oil refining industry [9]. The known external disturbance of the system has been used as a multivariable input, and the control structure given by using ideas from multivariable generalized predictive control [10]. The organic combination of feedforward control and feedback control has been realized, but this yields high computer hardware requirements. Li et al. [11] designed a control algorithm based on generalized predictive control for the extraction tower. Feng et al. [12] analyse the performance of a distributed model predictive control algorithm for a Fluid Catalytic Cracking (FCC) unit regenerator, and show that the distributed model predictive control algorithm has good control performance. Li et al. [13] consider a complex industrial process which is in series, with full consideration of the magnitude constraints, control incremental amplitude constraints, intermediate variable amplitude constraints, output error constraints and the final output amplitude constraints. Baldea et al. [14] consider a 
series process, develop a linear model predictive control algorithm and illustrate the reliability of the algorithm by simulation. Ma et al. [15] discuss energy saving by using distributed model predictive control. Minimizing the control objective is achieved by dynamic decoupling in the finite energy cost. The application of distributed predictive control in solar power generation is discussed in [16].

Distributed model predictive control is a new type of model predictive control method that has been increasing in popularity in recent years. This method takes into account the mutual communication among subsystems and thus greatly improves the control performance of the system. Given the condition that the hardware requirements of the controller must not be high, the decentralized model predictive control approach ensure the performance of the whole system is optimised. Therefore, it is an effective solution for the optimization and control of systems in series [17]. On the one hand, the general model predictive control and feed forward/feedback predictive control are used in the literature, but there are high performance requirements for the computer systems used for implementation. On the other hand, such hardware resources are seldom available in the atmospheric and vacuum tower of a petroleum refinery. This paper discusses the application of distributed model predictive control to the atmospheric and vacuum tower in order to seek a robust control solution that can be implemented in industry with reduced computation and complexity

This paper uses distributed model predictive control based on Nash optimality, and designs an optimal control for the atmospheric and vacuum tower in a petroleum refinery process. The objectives are to obtain good control performance, improve the robustness of the system, reduce the burden of online computation and reduce the cost of the control.

\section{THE ATMOSPHERIC AND VACUUM TOWER DYNAMIC MODEL}

In order to simplify the problem, this paper neglects control of the atmospheric furnace and vacuum furnace, and only studies atmospheric distillation and vacuum distillation. The whole system is assumed to be a series connection with two subsystems. Each subsystem has one input and one output. In the petrochemical industry, an important parameter is the socalled tray or plate efficiency. A tray or plate is a device that provides good contact between the vapour and liquid phases present in the distillation column.

To simplify the problem and without loss of generality, the following assumptions are made:

(1) The efficiency of the available Murphree liquid phase plate is assumed to be negligible;

(2) Each plate is well mixed and can be used as a centralized parameter system;

(3) The fluid mechanics of each plate can be expressed by the weir equation, and the instantaneous hydraulic gradient is established;

(4) Heat loss from the tower and the tower plate itself can be neglected and the reboiler and condenser heat transfer process will not be considered.
In accordance with the above assumptions, the dynamic equations will be established. A figure of the simplified physical model for the atmospheric and vacuum distillation tower follows to aid exposition:

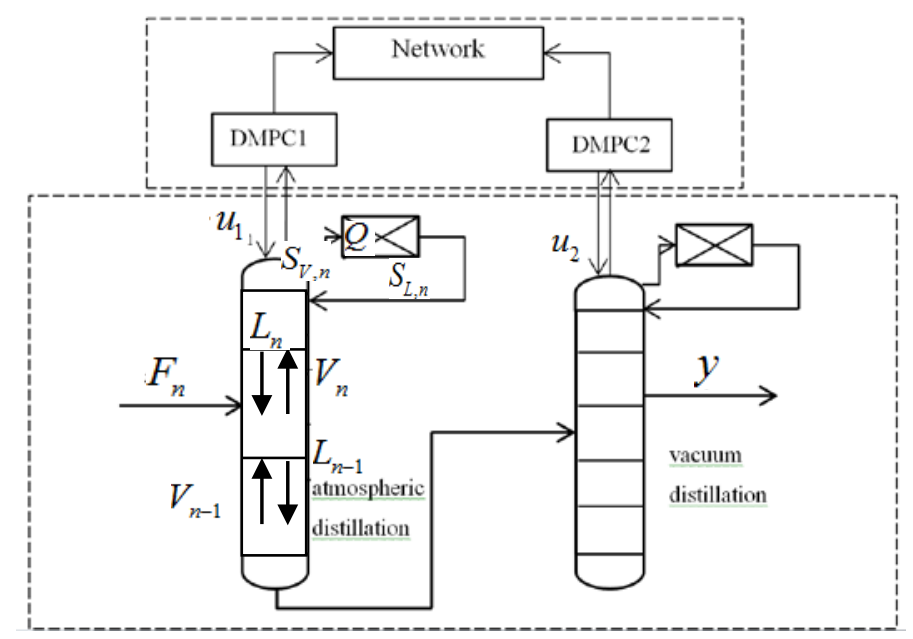

Fig. 1 Simplified physical model of the atmospheric and vacuum distillation towers

Here $u_{i}(i=1,2)$ denotes the control function, $Q$ is the heat load, $F_{n}$ is flow on the $n^{\text {th }}$ tray, $V_{n}, V_{n-1}$ respectively is the gas phase lateral line out of the material on the $n^{\text {th }}$ and $(n-1)^{\text {th }}$ tray, $L_{n}, L_{n-1}$ respectively is the liquid phase lateral line out of the material on the $n^{\text {th }}$ and $(n-1)^{\text {th }}$ tray, $S_{L, n}$ is the liquid phase reflux on the $n^{\text {th }}$ tray and $S_{V, n}$ is the gas phase reflux on the $n^{\text {th }}$ tray. From Fig 1, the input of the whole system is the input of the first subsystem (atmospheric distillation tower), and the output of the first subsystem is the input of the second subsystem (vacuum distillation tower). The equation of total material is given by

$$
\begin{gathered}
\frac{d M_{n}}{d t}=L_{n-1}+V_{n+1}+F_{n}-L_{n}-S_{V, n}-S_{L, n} \\
\frac{d M_{n} x_{i, n}}{d t}=L_{n-1} X_{i, n-1}+V_{n+1} y_{i, n+1}-\left(L_{n}+S_{L, n}\right) x_{i, n}+ \\
F_{n} x_{F_{i}, n}-\left(V_{n}+S_{V, n}\right) y_{i, n}
\end{gathered}
$$

where $M_{n}$ is the product discharge quantity on the $n^{\text {th }}$ tray. The heat balance equation yields:

$$
\begin{array}{r}
\frac{d M_{n} h_{n}}{d t}=M \frac{d h_{n}}{d t}+h_{n} \frac{d M}{d t}=L_{n-1} h_{n-1}+V_{n+1} H_{n+1}- \\
\left(L_{n}+S_{L, n}\right) h_{n}-\left(V_{n}+S_{V, n}\right) H+F_{n} h_{F, n}+Q_{n}
\end{array}
$$

where $H_{n}$ is the gas phase enthalpy on the $n^{\text {th }}$ tray and $h_{n}$ is the liquid phase enthalpy on the $n^{\text {th }}$ tray. 
The main function of the heat balance equation is to calculate $V_{n}$. The term $d h_{n} / d t$ cannot be ignored, otherwise significant errors result.

The weir equation, where the weir is used to promote the formation of vapour bubbles, is given by:

$$
L_{n}=C_{n} l_{n}^{\frac{3}{2}}
$$

where $L_{n}$ is the liquid layer; $l_{n}$ is the height of the overflow weir and $C_{n}$ is the coefficient of the weir.

The phase equilibrium is given by:

$$
y_{i, \mathrm{n}}=K_{i, n} x_{i, n}^{*}=K_{i, n} \frac{x_{i, n}-\left(1-\mathrm{E}_{i, n}\right) \mathrm{x}_{i, n-1}}{E_{i, n}}
$$

with the corresponding plate efficiency formula:

$$
E_{i, n}=\frac{x_{i, n}-x_{i, n-1}}{x_{i, n}^{*}-x_{i, n-1}}
$$

Synthesis of the physical and chemical properties enables a complete set of system dynamic equations to be formulated where the controlled variable is the back flow.

\section{DISTRIBUTED PREDICTIVE CONTROL ALGORITHM BASED ON NASH OPTIMALITY}

A distributed rolling solution is used whereby the control is calculated in a step-by-step fashion using rolling optimisation. A large-scale online optimization problem is replaced by a series of small scale distributed optimisation problems, which reduces the computational requirements. At the same time, due to the communication of information between the various elements, the adopted decentralized predictive control approach can overcome the performance degradation caused by the lack of information that may be expected from collapsing the scale of the problem considered for controller design. Using this approach, the overall control performance of the system can be maintained at a superior level [18].

Assuming that the whole system's behaviour can be seen as a result of the interaction of $N$ subsystems, the predicted output at the future time $P$ at the $k^{\text {th }}$ instant is:

$$
Y_{P M}(k)=f\left(Y_{P 0}(k), \Delta u_{1, M}(k), \ldots, \Delta u_{N, M}(k)\right)
$$

where

$$
Y_{P M}(k)=[Y(k+1 \mid k) \ldots \ldots(k+P \mid K)]^{T}
$$

$Y_{p 0}(k)=\left[Y_{0}(k+1 \mid k) \ldots \ldots . Y_{0}(k+P \mid k)\right]^{T}$ is the initial predicted value at the $k^{\text {th }}$ instant and $\Delta u_{1, M(k)}=\left[\Delta u_{i}(k) \ldots . . \Delta u_{i}(k+M-1)\right]^{T}$, where $N$ : control time domain, $P$ : prediction time domain, $f$ : mapping function vector. The constraint conditions for the system's output and input need to be satisfied as follows:

$$
\left\{\begin{array}{l}
\Delta u_{i, \min } \leq \Delta u_{i}(\bullet) \leq \Delta u_{i, \max } \\
u_{i, \min } \leq u_{i}(\bullet) \leq u_{i, \max }, \\
Y_{\text {min }} \leq Y(\bullet) \leq Y_{\max },
\end{array}\right.
$$

Define:

$$
\left\{\begin{array}{l}
\Delta u_{i}(k+j)=\Delta \mathrm{u}_{i}(k+M-1), \\
j=M, \ldots, P, i=1, \ldots, N
\end{array}\right.
$$

The performance index of the system is given by

$$
\min _{\Delta u_{1, M}(k), \ldots, \Delta u_{N, M}(\mathrm{k})} J=\sum_{j=1}^{P} L\left[Y(k+j \mid k), \Delta u_{1, M}(k), \ldots, \Delta u_{N, M}(k)\right] .
$$

The problem is distributed across the $N$ subsystems. Assuming that the performance index (3-4) is separable for the $N$ subsystems, the performance index of the $i^{\text {th }}$ subsystem can be taken as:

$$
\min _{\Delta u_{1, M}} J_{i}=\sum_{j=1}^{P} L_{i}\left[\mathrm{y}_{i}(k+j \mid k), \Delta u_{i, M}(k \mid k)\right] .
$$

The predicted output at the $k^{\text {th }}$ instant according to the formula (3-1) can be expressed as:

$$
y_{i, P M}(k)=f\left[\mathrm{y}_{i, P 0}(k), \Delta u_{1, M}(k), \ldots, \Delta u_{N, M}(k)\right] \quad(i=1, \ldots, N),
$$

where

$$
Y_{i, P M}(k)=\left[\mathrm{y}_{i}(k+1 \mid k) \ldots \ldots \mathrm{y}_{i}(k+P \mid K)\right]^{T} \quad \text { and }
$$

$Y_{i, P 0}(k)=\left[\mathrm{y}_{i, 0}(k+1 \mid k) \ldots \ldots \mathrm{y}_{i, 0}(k+P \mid k)\right]^{T}$.

The control problem can be solved with a Nash optimization method. Each subsystem optimises their own input variable with full knowledge of the input variables of the other subsystem.

$$
\min _{\Delta u_{i, M}(k)} J_{i} \mid \Delta u_{j, M}^{*}(k)(j=1, \ldots, N, j \neq i)
$$

$$
\begin{aligned}
y_{i, P M}(k)= & f_{i}\left[y_{i, P 0}(k), \Delta u_{1, M}^{*}(k), \ldots, \Delta u_{i-1, M}^{*}(k),\right. \\
\text { s.t. } \quad & \left.\Delta u_{i, M}(k), \Delta u_{i+1, M}^{*}(k), \ldots, \Delta \mathrm{u}_{N, M}(k)\right], \\
& \left\{\begin{array}{l}
\Delta u_{i, \min } \leq \Delta u_{i}(\bullet) \leq \Delta u_{i, \max }, \\
u_{i, \min } \leq u_{i}(\bullet) \leq u_{i, \max }, \\
y_{i, \min } \leq y(\bullet) \leq y_{i, \max } .
\end{array}\right.
\end{aligned}
$$

In this way, the Nash optimal condition of the whole system is as follows:

$$
\begin{aligned}
& J_{i}\left(\Delta u_{1, M}^{*}(k), \ldots, \Delta u_{i, M}^{*}(k), \ldots, \Delta u_{N, M}^{*}(k)\right) \leq \\
& J_{i}\left(\Delta u_{1, M}^{*}(k), \ldots, \Delta u_{i-1, M}^{*}(k), \Delta u_{i, M}(k), \Delta u_{i+1, M}^{*}(k), \ldots, \Delta u_{N, M}^{*}(k)\right)
\end{aligned}
$$

The network communication environment has a distributed structure where each subsystem can exchange information, so 
that the problem can be solved iteratively using the following steps:

Step 1: At the $k^{\text {th }}$ instant, each subsystem passes the initial control value to the other subsystems, and order $l=0$;

$\Delta u_{i, M}^{l}(k)=\left[\Delta u_{i}^{l}(k), \Delta u_{i}^{l}(k+1), \ldots, \Delta u_{i}^{l}(k+M-1)\right]^{T} \quad(i=1, \ldots, N)$

Step 2: Each subsystem solves its own predictive control problem (3-6) in parallel, and the optimal solution $\Delta u_{i, M}^{l+1}(k) \quad(i=1, \ldots, N)$ is obtained;

Step 3: Check whether the convergence condition of the predictor is satisfied for all subsystems, i.e., $\left\|\Delta u_{i, M}^{l+1}(k)-\Delta u_{i, M}^{l}(k)\right\| \leq \varepsilon_{i} \quad(i=1, \ldots, N) \quad$ for $\quad$ given $\varepsilon_{i}(i=1, \ldots, N)$. If so $\Delta u_{i, M}^{*}(k)=\Delta u_{i, M}^{l+1}(k) \quad(i=1, \ldots, N)$, end the iteration, and go to the fourth step. If not $\Delta u_{i, M}^{l}(k)=\Delta u_{i, M}^{l+1}(k) \quad(i=1, \ldots N), l=l+1$, and return to step 2 ;

Step 4: calculate the control at the $k^{\text {th }}$ instant, $\Delta u_{i}(k)=[I \ldots \ldots .0] \Delta u_{i, M}^{*}(k)(\mathrm{i}=1, \ldots, \mathrm{N})$;

Step 5: Scroll to the next time, that is $k+1 \rightarrow k$, return to step 1 and repeat the process.

The goal of this paper is to make the output of the system reach the expected value so that the fraction of oil achieving the desired goal reaches the set-point. This is achieved by controlling the flow rate, that is $u_{1}, u_{2}$, to control the valve opening of each subsystem to meet the overall performance index.

In this paper, there are two subsystems, the atmospheric distillation tower and vacuum distillation tower as described above. Decentralized model predictive control is used as outlined below. The predicted output of the whole system can be expressed as:

$$
Y_{P M}(k)=Y_{P 0}(k)+A \triangle u_{M}(k)
$$

The corresponding performance index is given by:

$$
\min J(k)=\left\|\omega(k)-Y_{P M}(k)\right\|_{Q}^{2}+\left\|\Delta u_{M}(k)\right\|_{R}^{2} .
$$

Here, $Q$ and are weight coefficient matrices, $\omega(k)$ denotes the desired output of the system, $\Delta u_{M}(k)=\left[\Delta u_{1, M}(k), \Delta \mathrm{u}_{2, M}(k)\right]^{T}$ and $A$ is the dynamic matrix. Using the distributed solution approach, and according to the model of the atmospheric and vacuum distillation towers previously presented, the whole system will be decomposed into two subsystems. Subsystem $1(i=1)$ is represented by:

$$
\begin{aligned}
y_{1, P M}(k)= & y_{1, p 0}(k)+A_{11} \Delta u_{1, M}(k)+ \\
& \sum_{\substack{j=1 \\
j \neq i}}^{2} A_{1 j} \Delta u_{j, M}(k) \quad(i=1, \ldots N) .
\end{aligned}
$$

where an equivalent subsystem can be written down for subsystem 2. On the right-hand-side of equation (3-10), $\sum_{\substack{j=1 \\ j \neq i}}^{2} A_{1 j} \Delta u_{j, M}(k)$ reflects the impact of the input of the other subsystems on the first subsystem. $A_{11}$ is the dynamic matrix of the first subsystem and the effect of the second subsystem on the step response matrix of the first subsystem is expressed by

$$
\begin{gathered}
A_{12}=\left[\begin{array}{cccc}
a_{12}(1) & \ldots & \ldots & 0 \\
\ldots & \ldots & \ldots & \ldots \\
a_{12}(\mathrm{M}) & \ldots & \ldots & a_{12}(1) \\
\ldots & \ldots & \ldots & \ldots \\
a_{12}(\mathrm{P}) & \ldots & \ldots & a_{12}(\mathrm{P}-\mathrm{M}+1)
\end{array}\right] \\
A=\left[\begin{array}{ll}
A_{11} & A_{12} \\
A_{21} & A_{22}
\end{array}\right]
\end{gathered}
$$

where $a_{12}(k) \quad(k=1,2, \ldots P)$ is the step response sequence. The performance index (3-10) is decomposed across the two subsystems, where the performance index of the subsystem 1 is given by:

$$
\min J_{1}(k)=\left\|\omega_{1}(k)-y_{1, P M}(k)\right\|_{Q_{1}}^{2}+\left\|\Delta u_{1, M}(k)\right\|_{R_{1}}^{2}
$$

In the formula, $\omega_{1}(k)=\left[\omega_{1}(k+1) \ldots \omega_{1}(k+P)\right]^{T}$ is the expected output value of the first subsystem with $Q_{1}$ and $R_{1}$ the corresponding weighting matrices.

At the initial instant, the real-time control rate is:

$$
\Delta u_{1}^{*}(\mathrm{k})=[I \ldots \ldots .0] \Delta u_{1, M}^{*}(k)
$$

Combining the algorithm steps, the next iteration of the optimal solution for subsystem 1 can be written as:

$$
\Delta u_{1, M}^{l+1}(k)=D_{11}\left[\omega_{1}(k)-y_{1, p 0}(k)-\sum_{\substack{j=1 \\ j \neq i}}^{2} A_{1 j} \Delta u_{j, M}^{l}(k)\right]
$$

where

$$
w(k)=\left[\omega_{1}(\mathrm{k}), \omega_{2}(k)\right]^{T}, y_{P 0}(k)=\left[y_{1, p 0}(k), y_{2, p 0}(k)\right]^{T}
$$

According to the above analysis, the iterative algorithm is convergent. The optimal solution of the entire distributed system can be written as:

$$
\Delta u_{1, M}^{l+1}(k)=D_{1}\left[\mathrm{w}(k)-y_{P 0}(k)\right]+\mathrm{D}_{0} \Delta u_{M}^{l}(k)
$$

where 


$$
D_{1}=\left[\begin{array}{ll}
D_{11} & D_{12} \\
D_{21} & D_{22}
\end{array}\right], D_{0}=\left[\begin{array}{cc}
0 & -D_{11} A_{12} \\
-D_{22} A_{21} & 0
\end{array}\right]
$$

This distributed optimization algorithm accommodates the influence of the other subsystems in every step of the rolling process and the Nash equilibrium can be reached for the system.

\section{SIMULATION RESULTS}

Based on the Nash optimal distributed predictive control algorithm developed in this paper, a simulation program is written in MATLAB and applied to the dynamic equation of the atmospheric and vacuum distillation tower series system. The performance of the proposed distributed predictive controller is shown in Fig. 2. Under the same conditions, a centralized model predictive control algorithm is adopted for
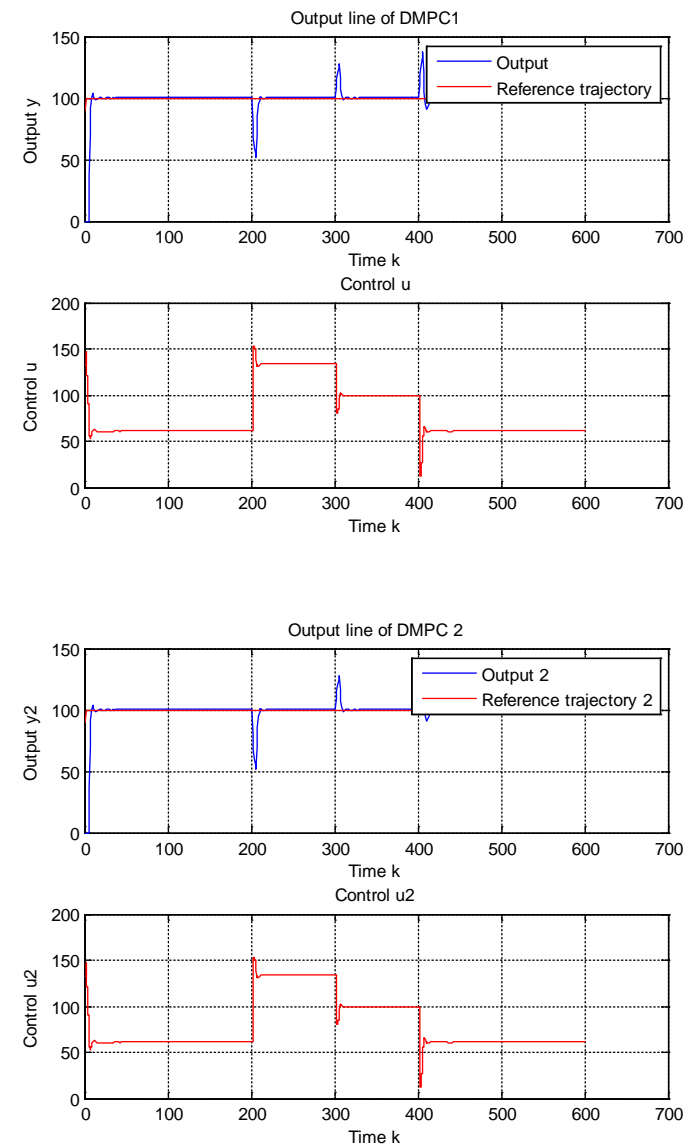

Fig. 2 Simulation results of DMPC for the atmospheric and vacuum distillation towers

comparison, and the results are shown in Fig. 3. A PID control algorithm is also adopted, and the simulation results are shown in Fig. 4.

From the simulation results, the case for applying an MPC strategy to this system is clearly made. The PID control strategy is insufficiently robust to tolerate the parameter uncertainty within the atmospheric and vacuum distillation tower as seen from Fig. 4. It can be seen from Fig. 2 that the distributed predictive control algorithm based on the Nash optimal controller fully considers the mutual communication between the two subsystems, so that each subsystem is well coordinated. The output curve exhibits a smooth rise with rapid regulation and limited overshoot. The system is clearly stable. The distributed predictive control algorithm achieves good overall performance.

When comparing Fig. 2 and Fig. 3, it is seen that in the distributed case, the performance of each subsystem can reach the same level as the centralized predictive control algorithm with the same control effort. However, the computation time of DMPC is 2.36s, while the computation time of CMPC is $3.87 \mathrm{~s}$. The DMPC obtains good performance with reduced computational effort when compared with the CMPC.
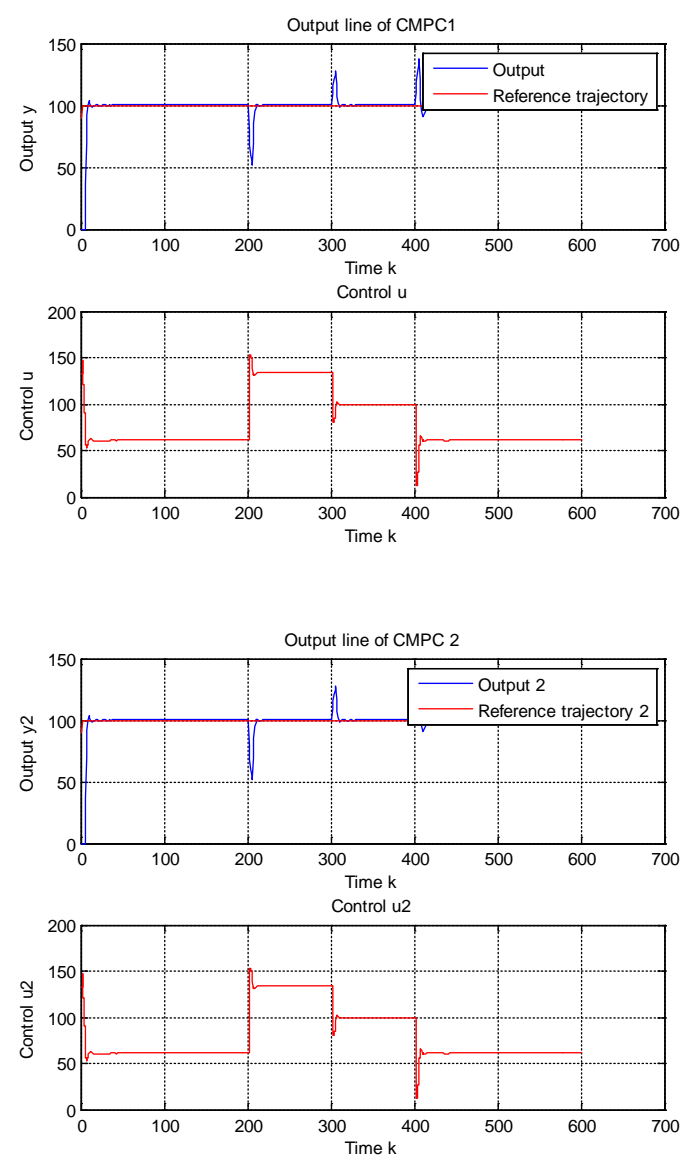

Fig. 3 Simulation results of CMPC for the atmospheric and vacuum distillation towers

Future work will conduct further studies including more elements within the refinery within the distributed control strategy with the ultimate objective of moving to practical implementation. 


\section{CONCLUSIONS}

In this paper, a distributed model predictive control algorithm based on Nash optimization is proposed for the atmospheric and vacuum distillation tower system within an oil refinery. The work adopts the characteristics of the network structure from the industrial environment. The methodology adopted can decompose a large-scale complex optimal control problem into several small scale distributed optimization problems. The overall performance as well as the performance of each subsystem can reach the same level as that achieved with a centralized predictive control algorithm. The level of control effort is seen to be the same in each case. At the same time, the distributed model predictive control approach reduces the computational burden involved in the implementation. This method is particularly suitable for complex cascaded systems such as the atmospheric and vacuum distillation tower. Future work will focus on the application of the distributed predictive control approach to more complex chemical industrial processes including implementation studies.

\section{ACKNOWLEDGMENTS}

This work is partially supported by the National Nature Science Foundation of China under Grant, 61273188, 61473312 and the Changjiang Scholars Program of China.
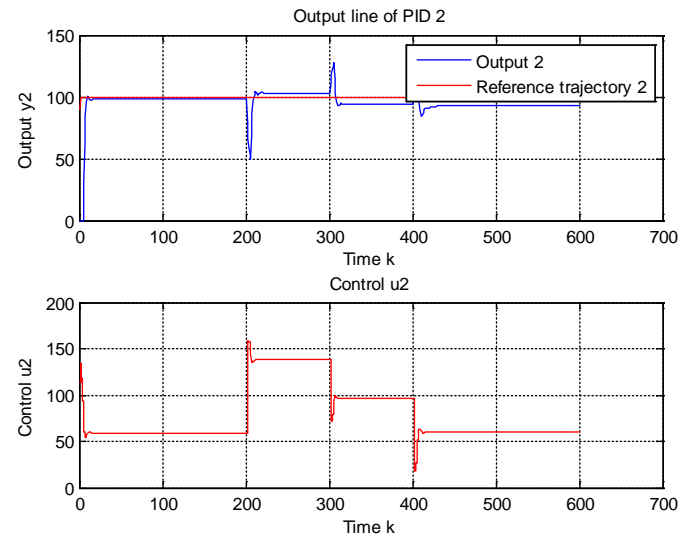

Fig.
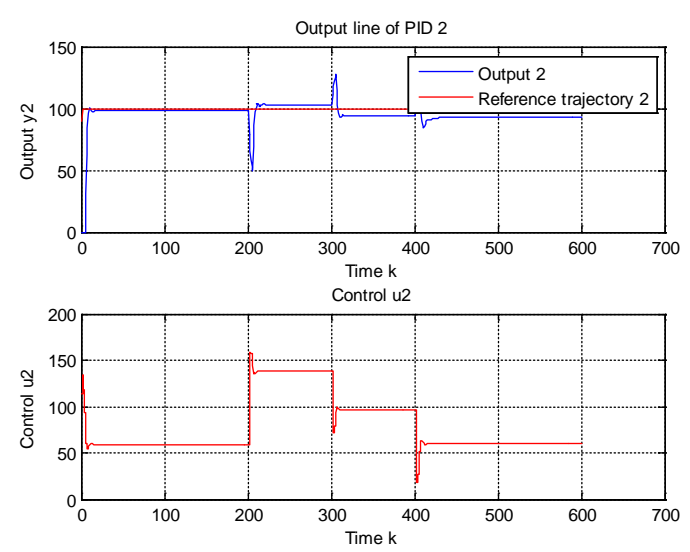

Fig. 4 Simulation results of PID control applied to the atmospheric and vacuum distillation towers

\section{REFERENCES}

[1] Wu, J., Zhang, L., and Chen, T.: 'Model predictive control for networked control systems’, Int. J. Robust Nonlinear Control, 2009, 19, (9), pp. 1016-1035

[2] Heemels, W.H., Teel, A.R., van de Wouw, N., Nesic, D.: 'Networked control systems with communication constraints: tradeoffs between transmission intervals, delays and performance', IEEE Trans. Autom. Control, 2010, 55, (8), pp. 1781-1796.

[3] Lee, J.H.: 'Model predictive control: review of the three decades of development', Int. J. Control Autom. Syst., 2011, 9, (3), pp. 415-424

[4] Camponogara, E. Scherer, H.F.: 'Distributed optimization for model predictive control of linear dynamic networks with control-input and output constraints', IEEE Trans. Autom. Sci. Eng., 2011, 8, (1), pp. 233242

[5] Shekhar, R.C., Maciejowski, J.M.: 'Robust variable horizon MPC with move blocking', Syst. Control Lett., 2012, 61, (4), pp. 587-594

[6] Ling, K.V., Maciejowski, J., Richards, A., Wu, B.F.: 'Multiplexed model predictive control', Automatica, 2012, 48, (2), pp. 396-401.

[7] Jerez, J.L., Ling, K.-V., Constantinides, G.A., Kerrigan, E.C.: 'Model predictive control for deeply pipelined field-programmable gate array implementation: algorithms and circuitry', IET Control Theory Appl., 2012, 6, (8), pp. 1029-1041.

[8] Rakovic, S.V., Kouvaritakis, B., Cannon, M., Panos, C., Findeisen, R.: 'Parameterized tube model predictive control', IEEE Trans. Autom. Control, 2012, 57, (11), pp. 2746-2761

[9] Xi Yugeng, Li Dewei, Lin Zhu. Modeling predictive control - status and challenges. Institute of Automation,2013,3(3):222 236

[10] Xi Yugeng. Predictive control, Defense Industry Press of Beijing, 1991.

[11] Li Qian, Li Ping, Hai Bin, Wang Shuqing. Multi feed forward and feedback generalized predictive control for series systems J. Control and decision, 2002, 7 (17): 500- 505.

[12] Feng Xu,Xionglin Luo, and Rui Wang. Design margin and control performance analysis of a fluid catalytic cracking unit regenerator under model predictive control, Control Engineering, 2014,7(11):79 81.

[13] Li Ping, Ren Penghui. Industrial series of multi-constrained systems generalized predictive control, Chemical Technology, 2010,8(61):2159 2164.

[14] Baldea, M., Daoutidis, P. \& Nagy, Z. K. (2010). Nonlinear model predictive control of integrated process systems. In Proceedings nonlinear control systems (NOLCOS 2010).

[15] Ma, Y., Kelman, A., Daly, A., Borrelli, F.: Predictive control for energy efficient buildings with thermal storage. IEEE Control System Magazine 32(1), 44-64 (2012)

[16] Yubin.Jia, X.J.Liu, . Distributed model predictive control of wind and solar generation system, C, Proceedings of the 33rd Chinese Control Conference, July 28-30, 2014, Nanjing, China . 7795-7799.

[17] Du Xiaoning, Xi Yugeng, Li Shaoyuan. Distributed predictive control optimization algorithm[J]. Control Theory and Applications. 2002,19(5):793 796.

[18] Chen Hong, Liu Zhiyuan, Xie Xiaohua. Nonlinear model predictive control status and problems,. Control and Decision, 2001,(16):385 391. 